\title{
Genomic screens identify a new phytobacterial microbe-associated molecular pattern and the cognate Arabidopsis receptor-like kinase that mediates its immune elicitation
}

\author{
G. Adam Mott ${ }^{1}$, Shalabh Thakur ${ }^{1}$, Elwira Smakowska², Pauline W. Wang ${ }^{3}$, Youssef Belkhadir², \\ Darrell Desveaux ${ }^{1,3^{*}+}$ and David S. Guttman ${ }^{1,3^{*}+}$
}

\begin{abstract}
Background: The recognition of microbe-associated molecular patterns during infection is central to the mounting of an effective immune response. In spite of their importance, it remains difficult to identify these molecules and the host receptors required for their perception, ultimately limiting our understanding of the role of these molecules in the evolution of host-pathogen relationships.

Results: We employ a comparative genomics screen to identify six new immune eliciting peptides from the phytopathogenic bacterium Pseudomonas syringae. We then perform a reverse genetic screen to identify Arabidopsis thaliana leucine-rich repeat receptor-like kinases required for the recognition of these elicitors. We test the six elicitors on 187 receptor-like kinase knock-down insertion lines using a high-throughput peroxidase-based immune assay and identify multiple lines that show decreased immune responses to specific peptides. From this primary screen data, we focused on the interaction between the xup25 peptide from a bacterial xanthine/uracil permease and the Arabidopsis receptor-like kinase xanthine/uracil permease sensing 1; a family XII protein closely related to two well-characterized receptor-like kinases. We show that xup25 treatment increases pathogenesisrelated gene induction, callose deposition, seedling growth inhibition, and resistance to virulent bacteria, all in a xanthine/uracil permease sensing 1-dependent manner. Finally, we show that this kinase-like receptor can bind the xup25 peptide directly. These results identify xup25 as a P. syringae microbe-associated molecular pattern and xanthine/uracil permease sensing 1 as a receptor-like kinase that detects the xup25 epitope to activate immune responses.
\end{abstract}

Conclusions: The present study demonstrates an efficient method to identify immune elicitors and the plant receptors responsible for their perception. Further exploration of these molecules will increase our understanding of plant-pathogen interactions and the basis for host specificity.

\footnotetext{
*Correspondence: darrell.desveaux@utoronto.ca; david.guttman@utoronto.ca

${ }^{\dagger}$ Equal contributors

'Department of Cell \& Systems Biology, University of Toronto, 25 Willcocks

St., Toronto, Ontario, Canada

Full list of author information is available at the end of the article
} 


\section{Background}

Effective immunity in plants relies upon a multi-tiered innate immune recognition system to successfully identify and appropriately respond to microbial invaders [1, 2]. This response requires the ability to quickly detect the presence of potential pathogens, effective mechanisms to disseminate that information through the organism, and finally appropriate physiological responses capable of controlling and clearing infection. The initial threat detection is largely accomplished through the recognition of microbe-associated molecular patterns (MAMPs), which are highly conserved immune elicitors derived from invading microbes. In the case of proteinaceous MAMPs, the genes encoding these microbial signatures are likely required for survival and, therefore, under strong negative selection as a whole [3]. If this were not the case, they would be modified or eliminated through natural selection in order to subvert host recognition. Nevertheless, while the genes encoding MAMPs are under strong negative selection overall, individual residues can show signals of positive selection for diversity [3]. This variation in what are otherwise conserved proteins may help the microbe avoid or dampen host recognition. In fact, MAMP sequence diversity has been shown to be associated with variation in the intensity of the immune response elicited by MAMP peptides [4-6].

The recognition of MAMPs is mediated through direct binding to a pattern-recognition receptor (PRR) on the plant cell surface. Characterized plant PRRs of peptide MAMPs contain an extracellular domain with a number of leucine-rich repeat (LRR) domains responsible for MAMP binding and can be divided based on their intracellular moieties into receptor-like kinases (RLK) and the closely related receptor-like proteins (RLP), which lack an intracellular kinase domain $[7,8]$. These two gene families in Arabidopsis thaliana have 223 and 57 LRR-containing members, respectively [9-11]; a level of genetic diversification suggestive of the key role these proteins play in plant evolutionary success. Immune signaling requires PRRs to work in complex with regulatory co-receptors, such as the Brassinosteroid Insensitive1-associated receptor kinase1 (BAK1) that regulates LRR-containing PRR activation $[12,13]$. This protein is an LRR-RLK that interacts with the PRR and appears to help shape the receptor pocket that binds directly to the MAMP $[12,13]$. While this co-receptor is not required for PRR-MAMP binding, it does participate in MAMP binding and both of these LRR-RLKs are required for full activation of immuneassociated receptor-like cytoplasmic kinases that transduce the immune activation signal.

The importance of MAMPs to plant immunity has led to considerable interest in their identification, yet surprisingly few MAMPs have been characterized. In fact, the majority of work on proteinaceous MAMPs to date has focused on flagellin and elongation factor $\mathrm{Tu}(\mathrm{EF}-\mathrm{Tu})$ and their respective eliciting peptides flg22 and elf18 [5, 6]. This is due in no small part to the technical challenges involved in the identification of both MAMPs and the plant components required for their perception using traditional means. More recently, with the proliferation of high-throughput sequencing and advances in bioinformatic techniques, predictive methods for MAMP identification have arisen. We have previously described such an approach based on the unique evolutionary signature of MAMP peptides that arises due to strong negative pressure to maintain protein function coupled to positive pressure to mutate sites in order to avoid plant perception [3]. While this early demonstration successfully identified peptides that elicited an immune response, it was not aimed at identifying the cognate host receptors required for recognition of these elicitors and consequently only addressed half of the issue. Another predictive approach has also recently been used to identify novel damage-associated molecular patterns (DAMPs) using sequence homology to known DAMPs [14]. Such approaches will become even more powerful as more genomes are sequenced and more immune elicitors are identified. Nevertheless, the host signaling components required for perception of these novel elicitors have yet to be identified.

The present study couples our computational pipeline for elicitor identification to a reverse genetic screen for cognate receptors using a high-throughput peroxidase assay. We show here both the identification of a novel Pseudomonas syringae MAMP that stimulates defence gene expression, induces callose deposition in planta, causes seedling growth inhibition, and functionally protects the plant from subsequent pathogen challenge, as well as the identification of an Arabidopsis thaliana LRR-RLK that binds the peptide and is required for the perception of this novel MAMP.

\section{Results \\ Computational identification of immune elicitors}

The screen for novel peptide elicitors was performed by examining $P$. syringae genomes for genes that show an overall pattern of strong negative selection for maintenance of required protein function, coupled to localized strong positive selection for avoidance of plant perception. We have used this approach previously to identify immune elicitors in P. syringae [3], but elected to generate a new set of candidates for this study due to the availability of vastly more genome sequences, which provided much more predictive power (see "Discussion" for more details). We examined $54 \quad P$. syringae isolates 
(Additional file 1: Table S1) to identify 3157 ortholog core gene families, as defined by being present in at least $90 \%$ of the isolates. A PAML selection analysis of this core gene set identified 172 genes that had at least one positively selected residue with $\omega$ ratio significantly greater than 1.0, and which rejected the neutral and negative selection null model (M7) in favor of the positive selection model (M8). Our previous study found that many putative immune elicitors had clusters of positively selected residues [3], therefore we used the Bayes Empirical Bayes estimates from the M8 model to narrow the list to those with clusters of at least three positively selected sites in a window of 25 residues, producing a candidate pool of 61 genes (Additional file 1: Table S2). This candidate pool included the wellestablished MAMPs EF-Tu and flagellin, ranking fifth and ninth, respectively, based on the number of predicted positively selected sites.

From the list of 61 positively selected core genes we identified a short-list of candidate peptides for this study based on: the level of significance of the PAML analysis; the overall strength of positive selection for the sequence; the number of positively selected residues clustered within a 25 residue window; the strength of selection acting on these clustered residues; and the likelihood of a false-positive signal due to the underlying alignment and proximity to the end of an assembly contig (see "Methods" for more details). We identified seven candidate 25 -amino-acid peptides for analysis, but later dropped one from the study due to inconsistent responses (Table 1 and Additional file 1: Table S3).

\section{High-throughput peroxidase immune elicitation assay}

We developed a sensitive, high-throughput, microtiterplate based screening assay based on innate immunityinduced peroxidase (POX) activity as a quantitative measure of immune function in order to efficiently screen large numbers of candidate elicitor peptides and LRR-RLK T-DNA insertion lines. The apoplastic POX enzymes play a role in the plant response to both wounding and pathogen infection [15-17]. These two responses, however, are temporally distinct, a fact that we used to avoid confounding our assay with woundinduced POX activity caused during tissue harvest. While POX activity has been used previously to study the effects of MAMP treatment [5], this is its first adaptation to a high-throughput screen using intact plant tissue. Importantly, the assay also showed excellent responses at a variety of doses of flg22, with sensitivity similar to the traditional ROS assay (Additional file 2: Figure S1).

The six candidate elicitors listed in Table 1 were tested for their ability to stimulate POX activity, and compared to the known MAMP flg22, a water-treated control, and a negative treatment consisting of an equimolar solution of a 25 amino acid peptide taken from a $P$. syringae gene present in the core genome that shows no evidence of positive selection. The four negative control peptides used are listed in Additional file 1: Table S4 and one member of the group was randomly assigned to each replicate experiment. These negative control peptides showed no immune elicitation in planta (Additional file 3: Figure S2) and are shown as a pooled single dataset for clarity where appropriate (these pooled data are marked as neg25). The six candidate peptides all elicited POX expression above the baseline (water control) and the neg25 negative control peptide level, although they were generally weaker elicitors than flg22 in A. thaliana ecotype Col0 plants (Fig. 1a). This level of elicitation for peptide elicitors is not unexpected, as flg22 is widely reported to be unique in the strength of its immune elicitation when compared to other peptide MAMPs and DAMPs $[3,14]$. We were, however, concerned with the possibility that our observed activity may be due to contaminating flg22 in our peptide preparations. In order to exclude this possibility, we also performed the POX assays in the A. thaliana ecotype Wassilewskija (WS) (Fig. 1b), which is insensitive to flg22 (Additional file 4: Figure S3) [18]. The activity of our peptides was maintained in WS, confirming that the activity arises from the novel peptide elicitors and is not due to contaminating flg22 peptide.

Table 1 Predicted peptide MAMPs

\begin{tabular}{|c|c|c|c|c|}
\hline Peptide name & Gene description & NCBI Gene ID & $P$ value ${ }^{a}$ & PSS $(\#)^{b}$ \\
\hline xup25 & Xanthine/uracil permease family protein & 1182392 & 1.13E-03 & 5 \\
\hline hyp25 & Conserved hypothetical protein & 1182322 & $1.60 \mathrm{E}-06$ & 12 \\
\hline isp25 & 4-diphosphocytidyl-2C-methyl-D-erythritol kinase & 1182741 & $3.13 \mathrm{E}-03$ & 8 \\
\hline atp25 & $A B C$ transporter, permease protein & 1182995 & $8.46 \mathrm{E}-03$ & 6 \\
\hline omp25 & Outer membrane protein & 1183357 & 2.91E-04 & 8 \\
\hline mpp25 & Membrane protein, putative & 1185283 & 1.99E-05 & 6 \\
\hline
\end{tabular}

${ }^{a} P$ value rejecting null hypothesis of neutral evolution

${ }^{\mathrm{b}}$ Number of positively selected sites 

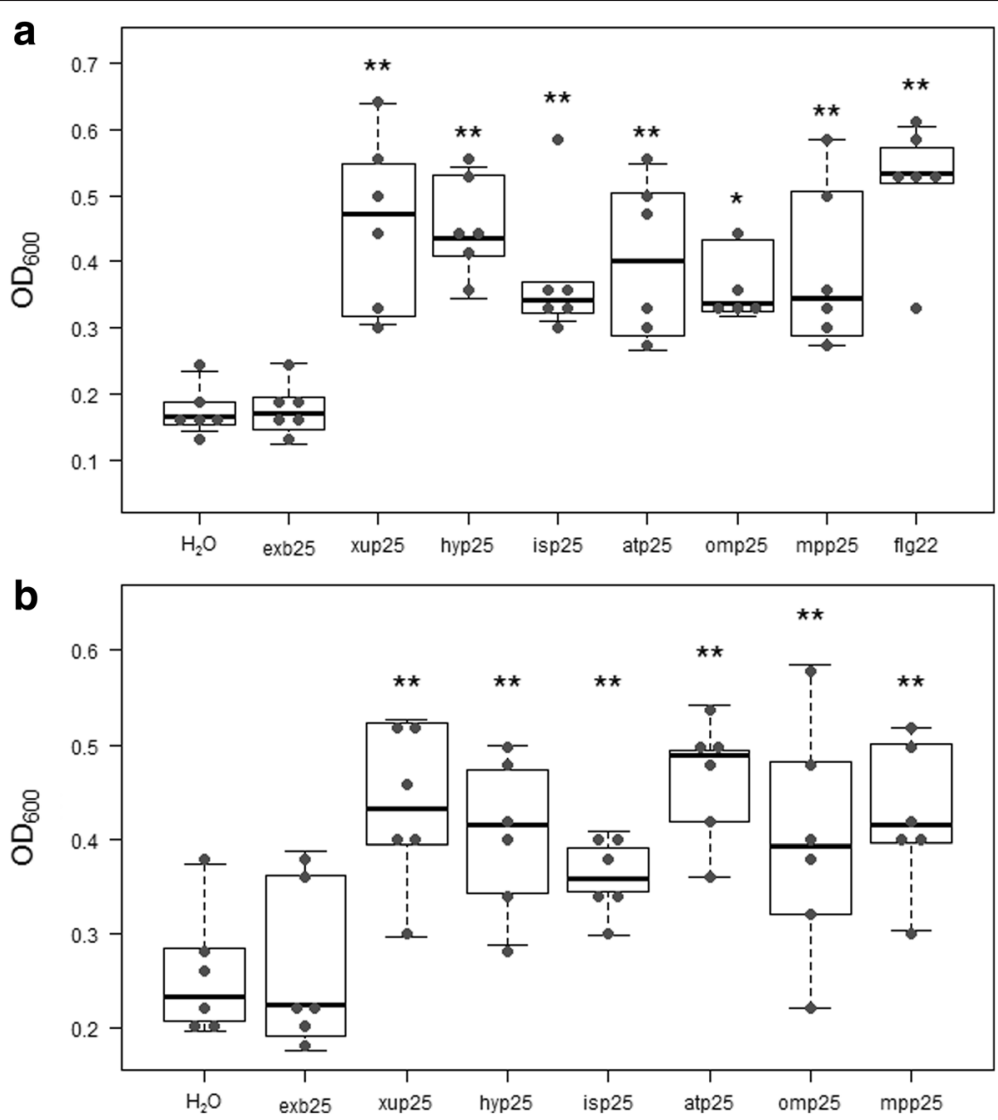

Fig. 1 MAMP treatment causes increased POX activity. Leaf disks from A. thaliana ecotype Col-0 (a) or Ws (b) plants were treated with water or $1 \mu \mathrm{M}$ of peptide and total POX activity was measured $20 \mathrm{~h}$ after treatment. Graphs are data from a single representative experiment $(n=6$, ${ }^{*} P<0.05,{ }^{* *} P<0.01,{ }^{* * *} P<0.001$, pairwise paired Student's $t$-test vs. the water control, corrected with Holm-Bonferroni). Closed circles represent individual observations, the boxes show the first quartile value, median value, and third quartile value, while the whiskers extend to the lowest and highest values in the data that are not deemed to be outliers (i.e. they are within $1.5 *$ IQR of the quartile value). All experiments were conducted a minimum of seven times with similar results

\section{Reverse genetic screen to identify MAMP receptors/co-receptors}

Having identified a set of elicitors that induced a robust and reproducible immune response in A. thaliana ecotypes Col- 0 and WS using the POX assay, we set out to identify plant LRR-RLKs required for the perception of these peptides. We identified a candidate list of $A$. thaliana ecotype Col-0 LRR-RLKs by searching the $A$. thaliana protein database for all proteins containing both an LRR and a kinase domain; recovering 227 proteins containing both domains, of which 216 also contain predicted transmembrane domains (Additional file 1: Table S5 and Fig. 2a, similar to previously published studies [9, 11]). We obtained 187 T-DNA insertional mutants representing 169 of the LRR-RLK encoding genes (several lines had T-DNA insertions in the same gene; Fig. 2a).

We performed a primary screen on the 187 T-DNA LRR-RLK insertion lines for their ability to respond to our six candidate elicitors using the high-throughput
POX assay. Each assay involved testing six replicate plants per genotype, with ten leaf cores per plant. All controls and peptides were assayed in parallel. Overall, the 187 T-DNA lines were assayed 3513 times (excluding negative controls), with a median of 496 assays per elicitor, 16.5 assays per T-DNA line, and 2.7 assays per elicitor per line.

Our aim for this study was to identify an LRR-RLK that was specifically required for mounting an immune response against a specific elicitor. We therefore focused on LRR-RLK T-DNA lines that were unable to respond to a single peptide, but which retained their ability to respond to flg22 and the other elicitors. Of the T-DNA lines that met this criterion, a line with an insertion in locus At3G47110 (hereafter XPS1) was of particular interest. XPS1 is evolutionarily closely related to the two best characterized peptide sensing LRR-RLKs: EFR and FLS2 $[19,20]$, as well as the $A$. thaliana ortholog of the rice LRR-RLK XA21, which recognizes a sulphated peptide from the RaxX protein of Xanthomonas oryzae pv. 


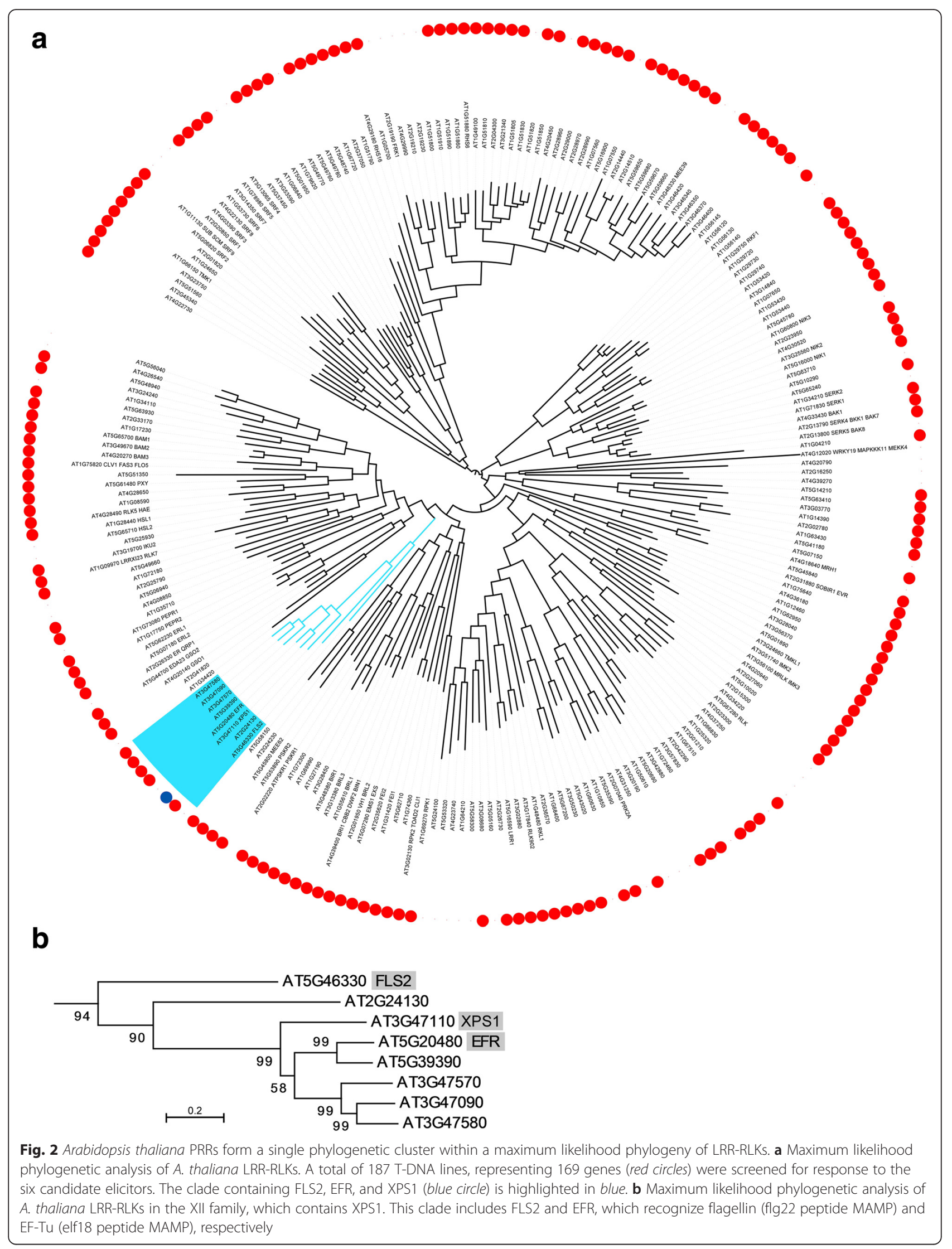


oryzae [21-24]. The clade containing XPS1, EFR, and FLS2 includes eight LRR-RLKs that form part of LRRRLK family XII (Fig. 2b) [11]. Importantly, two independent xps1 T-DNA lines responded normally to flg22 and the five other elicitors, while specifically losing sensitivity to xup25 (Fig. 3, Additional file 5: Figure S4, and Additional file 6: Figure S5). That xps1 retained flg22 sensitivity equal to that observed in Col-0 also reinforces that the original observed effect was not due to flg22 contamination of the xup25 peptide (Fig. 3).

\section{The XPS1 LRR-RLK is required for xup25-induced immunity}

MAMP treatment of $A$. thaliana results in many wellcharacterized responses, including inhibition of seedling growth [25], induction of defence gene expression [26], physiological changes including reinforcing the cell wall through callose deposition [25], and increased pathogen resistance [27]. To confirm our primary screen results, we first tested for the ability of xup25 to inhibit seedling growth in an XPS-dependent manner, a response that has been associated with immune activation [25]. We treated Col-0 and xps1 seedlings with flg22 and xup25 in liquid media, measured their subsequent growth, and found that the growth of Col-0 seedlings was significantly inhibited by both flg22 and xup25 peptides in a dose-dependent manner, while xps1 seedlings were growth-inhibited only by the flg22 peptide (Fig. 4).

The defence gene PR1 has been used extensively as a general marker of immune stimulation [25, 26]. We tested relative PR1 expression by treating Col-0 and xps1 plants with flg22 and xup25. Col-0 plants showed

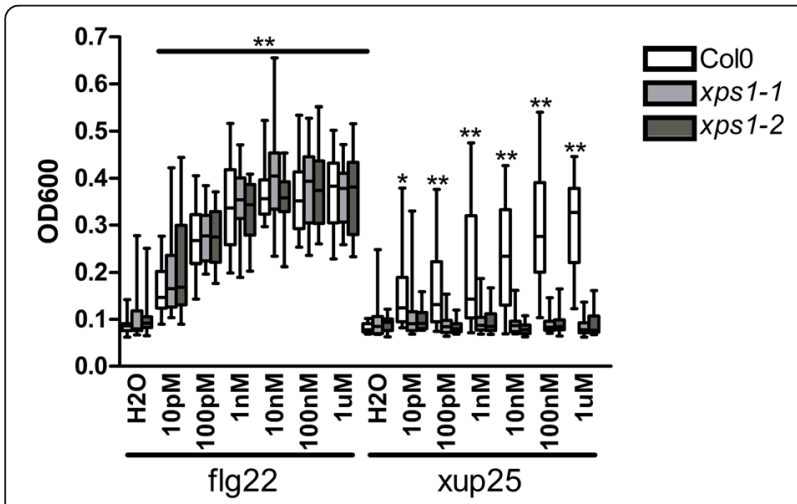

Fig. 3 XPS1 is required for POX induction by xup25. Leaf disks from A. thaliana ecotype Col-0 (white boxes), xps 1-1 (light gray boxes), or xps 1-2 (dark grey boxes) plants were treated with water or the noted concentration of flg22 or xup25 peptide and total POX activity was measured $20 \mathrm{~h}$ after treatment. Boxes show the lower quartile value, median value, and upper quartile value, while the whiskers extend to the lowest and highest values. Statistical comparisons are made between each treated sample and the untreated control of the same genotype ( $n=18$, ${ }^{*} P<0.05$, ${ }^{*} P<0.01$, repeated measures ANOVA followed by Dunnett's Test) strong PR1 induction to both treatments relative to the water control after $24 \mathrm{~h}$, while the xps 1 line responded only to the flg22 treatment (Fig. 5).

Another well-characterized marker of MAMP treatment is the deposition of callose to reinforce the plant cell wall [25]. We measured the levels of callose deposition following $24 \mathrm{~h}$ of treatment with flg22 or xup25. The treatment of Col-0 plants led to increased callose deposition in response to both peptides, whereas the xps 1 line showed callose deposition when treated with flg22 but not xup25, which showed no difference from the water control (Fig. 6 and Additional file 7: Figure S6).

The ultimate aim of the immune response is to increase pathogen resistance by suppressing colonization and growth of microbes in plant tissues. To measure elicitor-induced pathogen resistance, and to test the role of XPS1 in suppressing pathogen growth in response to xup25, we performed a virulence suppression assay by pre-treating plants with flg22 or xup25 peptides for $24 \mathrm{~h}$, and then challenging the plants with the highly virulent strain P. syringae pv. tomato DC3000 (Pto DC3000) via pressure infiltration. Plants that are pretreated with immune-eliciting peptides should show lower levels of pathogen growth. Both Col-0 and xps1 plants pre-treated with flg22 significantly suppressed in planta growth of Pto DC3000 24 h post infection (Fig. 7). In contrast, while xup25 pre-treatment was able to suppress Pto DC3000 growth in Col-0, it did not suppress pathogen growth in the xps 1 background, resulting in bacterial densities indistinguishable from the watertreated controls.

\section{XPS1 specifically binds xup25}

In order to demonstrate that XPS1 and xup25 represent a cognate MAMP-PRR pair, we next tested the binding of the peptide to the ectodomain of the receptor. For this, we expressed the extracellular domain of XPS1 in insect cells and purified it to near homogeneity (Fig. 8a). Subsequently, we used label-free microscale thermophoresis (MST) [28] to study the abilities of xup25 to bind to XPS1. As shown in Fig. 8b, XPS1 was able to bind the xup25 peptide with an observed EC50 of 103 $\mathrm{nM} \pm 0.048$ at an MST power of $60 \%$. We repeatedly performed our measurements on two independent protein preparations to ensure reproducibility. Our second assay at the same MST power displayed a similar binding profile, yet showed an increased EC50 (381 nM \pm 0.054). A control experiment in which we tested the ability of XPS1 to bind the flg22 peptide demonstrated the specificity of the xup25-XPS1 interaction (Additional file 8: Figure S7). Thus XPS1 can discriminate unrelated peptides and our binding assays strongly indicate that XPS1 is directly involved in the perception of xup25. 


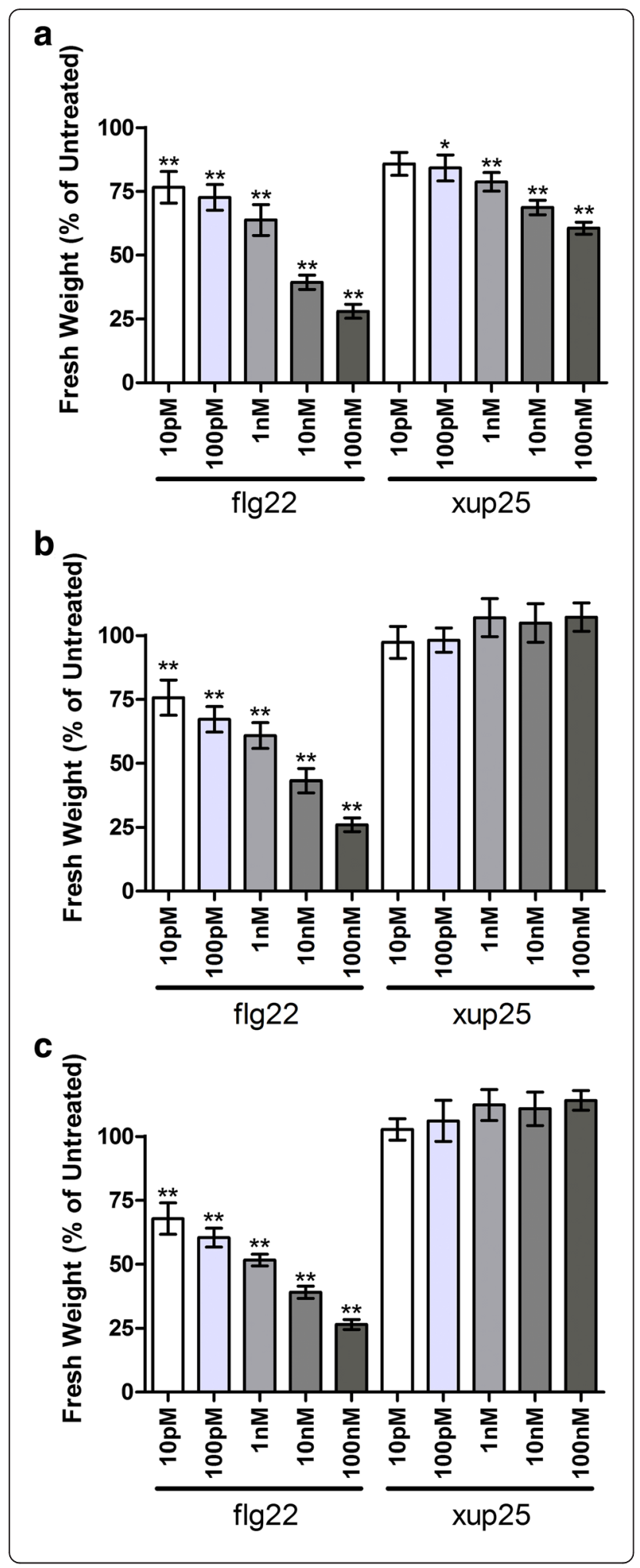

Fig. 4 xup25 inhibits seedling growth in an XPS1-dependent manner. Five-day-old seedlings of A. thaliana ecotype (a) Col-0, (b) xps 1-1, or (c) xps 1-2 were grown for a further 10-14 days in liquid MS media containing water or the noted dose of peptide. The seedlings were removed, briefly dried, weighed (fresh weight), and those weights normalized to the mean weight of the control sample. As least four seedlings of each treatment were included and the entire experiment replicated at least three times and all data are presented. Statistical comparisons are made between each treated sample and the untreated control of the same genotype $\left(n \geq 12,{ }^{*} P<0.05\right.$, ${ }^{* *} P<0.01$, one-way ANOVA followed by Dunnett's Test)

\section{Discussion}

In spite of the important role that MAMPs play in plant immunity, only a small number have been identified, and an even smaller number of proteinaceous MAMPs have been linked to specific LRR-containing receptors. The traditional approaches for MAMP identification rely upon refining complex mixtures of molecules from pathogens, which becomes increasingly difficult when working with MAMPs that have weak biological effects under specific test conditions. In addition, our experimental models can only crudely mimic the complex milieu of microbial commensals, mutualists, and parasites that constantly interact with plants in the real environment. Interactions that we perceive as weak under rarefied laboratory conditions may in fact be extremely important under specific ecological conditions. While these ecological and evolutionary processes normally limit

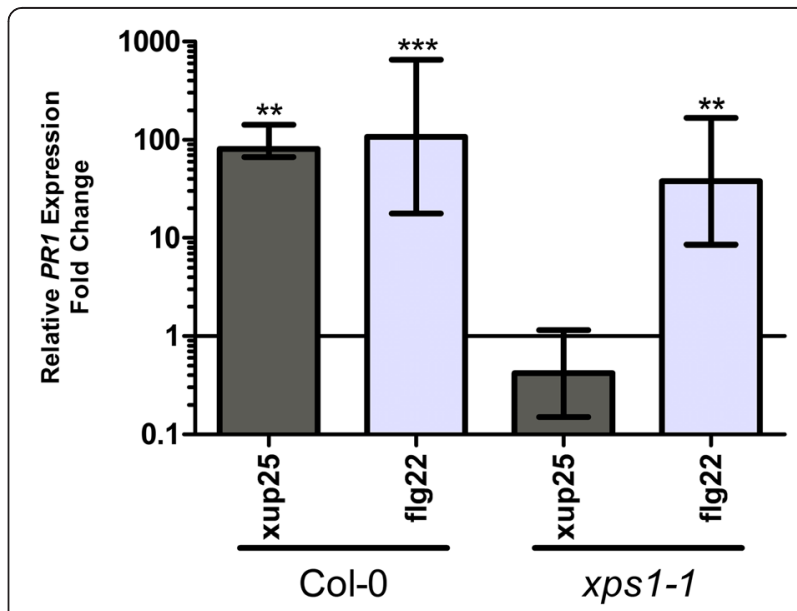

Fig. 5 xup25 induces defence gene expression in an XPS1-dependent manner. Quantitative real-time PCR analysis of the relative expression of the defence gene PR1 in Col-0 and xps 1-1 A. thaliana plants after $24 \mathrm{~h}$ of treatment with water or $10 \mu \mathrm{M}$ of peptide normalized to UBQ10 expression. Tissue from two individuals for each treatment were pooled prior to RNA harvest. Bars represent mean values $+/$ - s.e. from three biological experiments each assayed in triplicate. Statistical analysis was carried out on dCt values, comparing treatments to water treated control samples using pairwise student's t-tests with Holm-Bonferroni correction ( ${ }^{* *} P<0.01$, ${ }^{* * *} P<0.001$ ). No significant difference was observed between Col-0 and xps 1-1 plants treated with flg22 


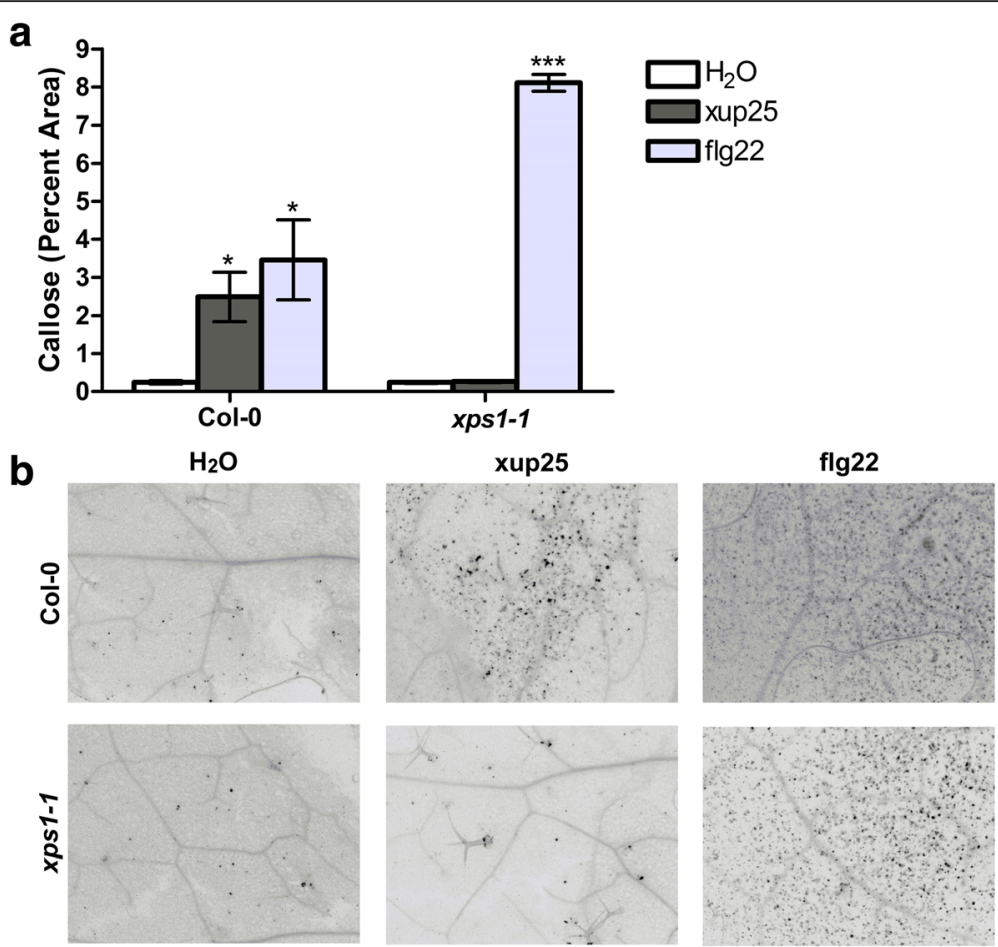

Fig. 6 xup25-induced callose deposition is XPS1-dependent. Leaves from A. thaliana ecotype Col-0 or xps 1-1 plants were pressure infiltrated with water or $10 \mu \mathrm{M}$ of peptide. After $24 \mathrm{~h}$ of treatment the leaves were harvested, cleared, and callose deposits were stained prior to epifluorescent microscopy. a The proportion of the image with callose present was determined and data from a single representative trial is shown ( $n=6$, ${ }^{*} P<0.05$, ${ }^{* *} P<0.001$, pairwise Student's $t$-test, corrected with Holm-Bonferroni). b Representative images of the callose deposits for each treatment are shown. Callose deposits are indicated by dark spots; the images have been converted to gray scale and inverted. The experiment was repeated four times in total with similar results, for a total $n=26$

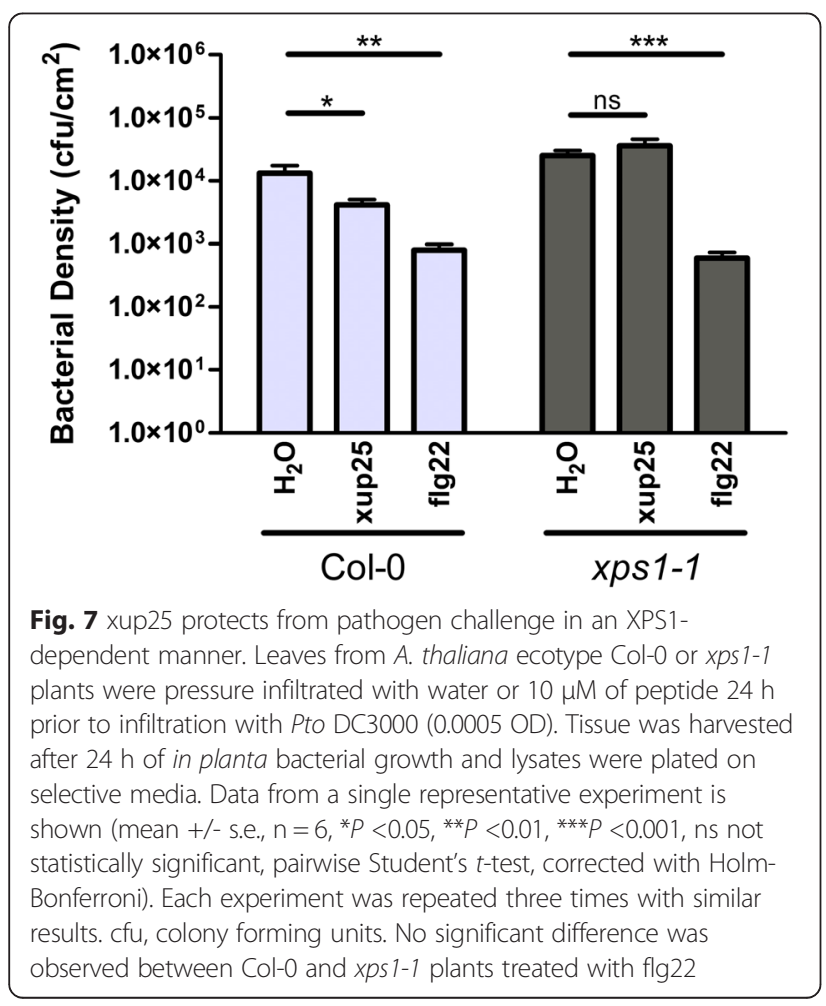

progress, our computational approach to MAMP identification instead takes advantage of these events by mining for the genetic footprints left by these interactions.

This study used comparative genomics analyses to identify peptides carrying genetic signatures consistent with expectations for MAMPs. Six of these immuneeliciting peptides were used in a reverse genetic screen against 187 A thaliana T-DNA insertion lines of putative PRRs. While most of the knockout lines retained their ability to mount an immune response when challenged with the peptides, one line, xps1, was unable to mount any measurable immune response to the xup25 peptide. Importantly, the xps 1 line retained its ability to respond to the other peptides, including flg22. Finally, the XPS1 ectodomain is shown to be involved in the direct binding of xup25.

These results indicate that XPS1 can bind to xup25 and is specifically required for perception and, therefore, xup25 and XPS1 can be considered a MAMP and PRR pair in the strictest sense.

The xup25 peptide is derived from a xanthine/uracil permease family protein (conservation is shown in Additional file 9: Figure S8). There is no clear reason why this protein should be a source of a peptide MAMP 

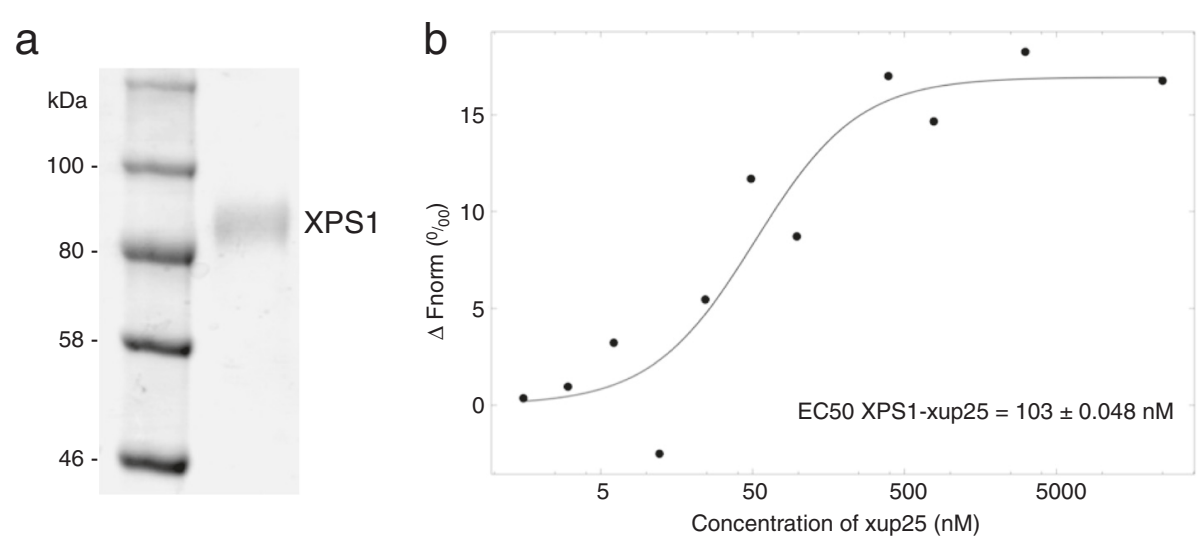

Fig. 8 XPS1 binds specifically to xup25. a Coomassie stain of purified XPS1 ectodomain used in MST assays. b Quantification of binding between the XPS1 ectodomain and xup25 by label-free MST. The XPS1 ectodomain was kept at a constant concentration (0.5 $\mu$ M) whereas varying peptide concentrations were added. Data points indicate the difference in normalized fluorescence (\%) generated by xup25 binding to the XPS1 ectodomain. Curves are plots of xup25 concentrations against percent changes of normalized fluorescence ( $\Delta$ Fnorm [\%] $y$-axis). Curve fitting was performed by using the NT affinity analysis software from Nanotemper. The EC50 value calculated at MST power of $60 \%$ is indicated on top. The binding profile is representative of two independent assays performed with two independent protein preparations (see Additional file 9: Figure S8). The standard error of the regression fit is 2.866732

beyond the fact that it is highly conserved. In fact, all six candidate elicitors identified in this study are broadly conserved among the bacteria, while some are even found in the archaea (data not shown). Four of the candidates, including the protein encoding xup25, are membrane proteins, which while not a requirement for a MAMP (q.v. EF-Tu), may increase the likelihood that the peptides come in contact with host PRRs.

The amplitude of the immune response we observe with the newly identified peptide elicitors is lower than that observed for flg22. Recent work has shown that natural variation within a MAMP sequence can have large effects on the resulting immune response [4, 29], raising the question of whether we would have observed stronger immune responses if we had designed our peptides based on protein sequences from more divergent bacteria with non-host interactions, rather than $P$. syringae. As there has not yet been a broad survey of the relationship between MAMP origin and the strength of immune elicitation, we can only speculate on the matter based on the limited data available. In the best studied case, the flg22 peptide from Pseudomonas aeruginosa elicits a stronger immune response in A. thaliana than the corresponding peptide from $P$. syringae [30]. This finding may seem logical, as $P$. aeruginosa is a non-host species rarely found on foliar surfaces, suggesting flg22 may be at least partially responsible for this niche restriction. Upon closer examination, this theory is contradicted by the fact that the flg22 peptide does not elicit an efficient immune response in all non-host plant species studied (e.g. celery [29]), nor do all natural accessions of $A$. thaliana carry the FLS2 PRR that recognizes flg22 [18].
In fact, the opposite argument could also be made, namely that MAMPs from pathogens of a specific plant host should elicit a strong immune response, since these are ecologically relevant interactions. This argument is supported by the observation that FLS2 restricts the growth of even highly virulent pathogens such as Pto DC3000 [31]. While type III secreted effectors also strongly influence disease and immunity, these proteins are highly variable even among closely related strains [32-34] and, consequently, likely mediate a relatively small number of all plant-microbe interactions. We elected to test candidate elicitor peptides derived from the host strain Pto DC3000, as well as a number of homologous peptides derived from the non-host strain P. syringae pv. phaseolicola 1448a, but found no significant differential response (data not shown). Future work examining homologous peptides from a wider range of species will help inform which of these two positions is more generally correct.

Previous work in our group has used a similar bioinformatics approach to identifying immune eliciting peptides [3], but the current report represents a significant improvement in several respects. Most notably, the previous work was performed when only three P. syringae isolates had been sequenced, necessitating the inclusion of three strains of Xanthomonas campestris to achieve sufficient sample size for the selection analyses. The inclusion of these divergent genomes strongly influenced the prediction of the set of core genes. Additionally, the use of two quite divergent species groups negatively influenced the quality of the alignments and ultimately the predictions of positive and negative 
selection as gap positions are ignored in PAML selection analyses. In the earlier report, we characterized the immune eliciting properties of eight bioinformatically predicted peptides. The proteins from which these peptides were derived were also analyzed as part of this report, however the use of expanded genome data from $54 P$. syringae strains resulted in our decision to select new candidates for a variety of reasons. Three of the previously identified proteins (PSPTO2757, PSPTO3620, and PSPTO4436) were not present in a sufficient number of strains to be considered part of the core genome in this report. This observation may be true or simply an artefact due to the extensive use of incomplete draft genomes in the current study. Of the remaining five candidates from the previous report, four had positively selected sites (PSPTO3468, PSPTO0175, PSPTO5086, and PSPTO0624), while the last did not show a significant signal of positive selection in the new analysis (PSPTO1253). The inclusion of PSPTO1253 was unique in the previous report as it was based on the presence of a single PSS, making it the least robust prediction of the set. We elected not to use the four previously identified peptides that were also present in this new study since the expanded dataset and subsequent refined analysis identified other candidates with stronger signals based on the number and clustering of positively selected sites. The current analysis of 54 P. syringae genomes provides better sequence alignments, a much more reliable prediction of the species-specific core genome, greatly enhanced statistical power to detect signatures of positive selection, and ultimately a more robust prediction of MAMP candidates.

XPS1 is notable as it forms a part of the LRR-RLK family XII along with FLS2 and EFR, the receptors that recognize the peptide MAMPs flg22 and elf18, respectively $[19,20]$. This clade also contains the $A$. thaliana ortholog of the rice LRR-RLK XA21 (based on reciprocal BLAST analysis), which recognizes a sulphated peptide from the RaxX protein to confer resistance to Xanthomonas oryzae $\mathrm{pv}$. oryzae [21, 22, 24]. In addition to the phylogenetic proximity, the presence of a predicted 23 LRR domains (data not shown) in the XPS1 extracellular domain suggests the protein acts as a receptor. It has previously been suggested [35] that the number of LRR domains present in a given LRR-RLK can be used to predict whether that protein functions as a co-receptor, such as BAK1 with only five LRR domains $[36,37]$, or receptor, such as FLS2 or EFR which have 28 and 21 LRR domains, respectively $[19,20]$. These observations suggest that the clade of LRR-RLKs containing FLS2, EFR, and XPS1 may be involved generally in MAMP perception and provides several excellent candidates for further inquiry.

There are few previous examples of studies that quantitate the binding affinity between a PRR and its peptide
MAMP. While the techniques used in those reports differ from those used here, they generally report $\mathrm{IC}_{50}$ values in the low $\mathrm{nM}$ range $[18,20,38]$. While not directly comparable, the apparent EC50 of XPS1 for xup25 is in the high nM range. Our attempts to fit our data using a dissociation constant $\left(\mathrm{K}_{\mathrm{D}}\right)$ model showed highly significant variation between the two assays we performed (using independent protein preparations) even at different MST powers. Therefore, we were not able to calculate an accurate overall $\mathrm{K}_{\mathrm{D}}$ for the XPS1-xup25 binding. We attribute this variation in $K_{D}$ to nonoptimized binding events driven by the heterogeneous $\mathrm{N}$-glycosylation of the XPS1 ectodomain in our expression system (as observed in coomassie stained samples of the purified protein (Fig. 8a)). Alternatively, the variations in binding constants might reflect the use of a non-optimized xup25 peptide sequence in our binding assays. In this respect, we anticipate that interaction assays involving shorter or longer variants of xup 25 will be very informative. Additionally, while we definitively show that the XPS1 binds to xup 25 and is required for its biological activity, this does not eliminate the possibility that the peptide binds to other cell surface molecules including co-receptors. A missing required co-receptor in these in vitro binding assays would also explain the observed variability and current efforts in the lab are focused on discovery of other members of the binding complex.

To date, the identification of PRRs has relied primarily on forward genetic screens, with limited examples of successful reverse genetics approaches $[14,20,39]$. While this forward genetics approach has been very successful, it is limited when applied to MAMPs with weak biological effects or less robust phenotypes. We instead employed a reverse genetics approach to screen the predicted elicitors against a collection of predicted $A$. thaliana immune receptors. This method required a collection of LRR-RLK knockout lines and the use of a high-throughput assay to measure immune elicitation (the POX assay), but enabled us to screen a wide diversity of candidate immune receptors. While most LRRRLK T-DNA lines showed a wild-type response to all tested peptides, some displayed general hyper- or hyposensitivity to the suite of elicitors, while a smaller group (including xps1) showed specific loss of sensitivity to a single peptide; thereby providing a wealth of leads for the dissection of plant immune signaling pathways.

As genomic and transcriptomic data become more available, these bioinformatics approaches to MAMP and PRR identification will become even more powerful. The continued elucidation of the true range of MAMP epitopes and their cognate receptors will not only benefit plant health, but also increase our understanding of the evolution of the plant-pathogen interaction. 


\section{Conclusions}

Here we expand on our previous work, utilizing a predictive bioinformatics approach to identify potential MAMPs based on their unique evolutionary signatures, and then use these immune elicitors in a reverse genetic screen to identify their putative receptors or coreceptors. We show that the xup25 peptide derived from a xanthine/uracil permease protein is a novel MAMP with the associated biological effects, including plant growth inhibition, induction of defence gene expression and callose deposition, and the stimulation of a functional immune response sufficient to suppress the growth of a pathogen. Most significantly, xup25 specifically requires the function of the LRR-RLK XPS1 to elicit all of these responses and directly binds to this plant receptor. While we have focused on one particular interaction to validate the methodology, both the computational MAMP screen and the subsequent reverse genetic receptor screen have provided numerous candidates for future study. Of particular interest are those LRR-RLKs that show consistent patterns of hypo- and hypersensitivity to multiple elicitors. These proteins may play key roles in regulating the $A$. thaliana basal immune response, and therefore, are potentially important targets for the development of resistant crops.

\section{Methods}

\section{Plant materials and growth conditions}

All plants were grown with a 12 -h photoperiod (100$150 \mu \mathrm{mol} / \mathrm{m}^{2} \mathrm{~s}$ ) at $21{ }^{\circ} \mathrm{C}$, followed by a 12-h dark period at $20{ }^{\circ} \mathrm{C}$ in a Conviron growth room (Conviron). All T-DNA lines were selected using the TDNA Express database of the Salk Institute Genomic Analysis Laboratory (SIGnAL, http://signal.salk.edu/ cgi-bin/tdnaexpress). Preference was given to lines with insertions in coding sequences. The genotypes of lines of interest were confirmed by PCR using primers designed using the T-DNA primer design tool (http://signal.salk.edu/tdnaprimers.2.html).

\section{Core-genome prediction}

The analysis was performed using protein sequences from the genomes of $54 \mathrm{P}$. syringae strains. The dataset included genomes from 28 strains available in public databases and 26 strains sequenced by the University of Toronto Centre for Analysis of Genome Evolution and Function (Additional file 1: Table S1). The ortholog prediction was performed using the OrthoMCL program with protein blast e-value $1 \mathrm{e}-10$ [40]. The protein sequences were predicted as ortholog pairs if they shared at least $70 \%$ identity over $70 \%$ sequence length. OrthoMCL was used to cluster ortholog pairs into the ortholog protein families using a markov chain clustering algorithm.
The ortholog families that were present in at least $90 \%$ of the strains were defined as core gene families.

\section{Selection analysis}

The codon-based multiple alignments were constructed with translated protein sequences using translatorX and the MUSCLE multiple alignment program [41, 42]. The protein-based phylogenetic tree was built for each core gene family using the FastTree program [43]. The patterns of natural selection were detected in the predicted core gene families by using the codeml program in the PAML application suite [44]. The prediction of positive selection in the core genes was performed by applying codeml random-site models M7 (neutral selection) and M8 (positive selection) that allow the $\omega$ ratio $\mathrm{dN} / \mathrm{dS}$ to vary among sites. A likelihood ratio test was used to reject the neutral and negative selection null model (M7) in favor of positive selection model (M8) with the chisquare $P$ value $<0.01$ for all candidate core genes. Empirical Bayes estimates from the model M8 giving a posterior probability $>0.5$ were used to identify positively selected codons.

\section{Peptides}

The predicted peptide MAMPs were designed from the $P$. syringae Pto DC3000 genome sequence as 25 amino acid sequences. The flg22 sequence is taken from the $P$. aeruginosa genome sequence as a 22 amino acid sequence. All peptides were purchased from the Sheldon Biotechnology Centre at McGill University and GenScript.

\section{Peroxidase assay}

Single leaves were excised from six plants per genotype of mature $A$. thaliana plants. From each leaf ten size one leaf cores were taken and washed for $1 \mathrm{~h}$ in $1 \mathrm{~mL}$ of 1X MS solution with agitation. After washing, leaves were transferred to individual wells of a clear 96-well assay plate avoiding the use of the edge wells to minimize evaporation effects. Each well received $50 \mu \mathrm{L}$ of $1 \mathrm{X}$ MS buffer alone or supplemented with $1 \mu \mathrm{M}$ of each peptide. Thus each leaf was tested with each treatment, allowing for paired statistical testing. Plates were sealed with parafilm and incubated for $20 \mathrm{~h}$ with agitation. The leaf disks were removed and each well received $50 \mu \mathrm{L}$ of a $1 \mathrm{mg} / \mathrm{mL}$ solution of 5 -aminosalicylic acid (A79809, Sigma-Aldrich) pH 6.0 with $0.01 \%$ hydrogen peroxide. The reaction was allowed to proceed for 1$3 \mathrm{~min}$ and stopped by the addition of $20 \mu \mathrm{M} 2 \mathrm{~N} \mathrm{NaOH}$ prior to reading the $\mathrm{OD}_{600}$ on a POLARstar OPTIMA microplate reader (BMG Labtech).

\section{LRR-RLK prediction and alignment}

RLKs were identified by identifying all proteins with both an LRR domain similar to the LRR domain of 
either FLS2 or EFR and a kinase domain similar to the kinase domain of FLS2. Similarity searches were performed using both Delta BLAST [45] and PSI-BLAST [46] with the respective conserved domain. The A. thaliana RefSeq database was queried using a minimum Evalue threshold of $1 \mathrm{e}-08$, and the searches were iterated until no new sequences were added. RLK candidate proteins were selected for further analysis only if they had a match to both an LRR domain and a kinase domain, resulting in 227 candidates. Global multiple sequence alignment was performed with the MAFFT E-INS-i algorithm [47] and a maximum likelihood phylogeny was constructed in MEGA v5 [48] using partial deletion and $25 \%$ site coverage cutoff, the WAG with Freqs. (+F) substitution mode, gamma distribution with five rate categories, and 100 bootstraps.

\section{Confirmation of T-DNA insertion lines}

The SALK lines SALK_101647 (xps1-1) and SALK_ 101668 (xps1-2) were obtained from the Arabidopsis Biological Resource Center (ABRC) [49]. RT-PCR analyses were performed to verify alterations in transcript levels. Plant RNA was extracted from leaves, which were excised, pooled, and flash frozen in liquid nitrogen prior to homogenization by mortar and pestle under liquid nitrogen. Total RNA was extracted using TRI Reagent as per manufacturer's instructions (SigmaAldrich), followed by DNaseI treatment (Thermo Scientific). A total of $2 \mu \mathrm{g}$ of RNA was used for cDNA synthesis according to the manufacturer's instructions with the SuperScript II reverse transcriptase (Life Technologies). Amplification of UBQ10 served as an internal control in RT-PCR assays. The PCR primers used were: XPS1 LRR domain 5'- TCTTCACTAA TATTCCTG-3' and 5'- TGATAGTTTATTGTATGA-3', UBQ10 (At4g05320) 5'-CACACTCCACTTGGTCTTG CGT-3' and 5'-TGGTCTTTCCGGTGAGAGTCTTCA-3'. The PCR included 35 cycles for XPS1 LRR domain, and 32 cycles for $U B Q 10$.

\section{Real-time PCR assay}

A single leaf on two individual plants was pressure infiltrated with water alone or supplemented with $10 \mu \mathrm{M}$ peptide. After $24 \mathrm{~h}$ of treatment the leaves were excised, pooled, and flash frozen in liquid nitrogen prior to homogenization by mortar and pestle under liquid nitrogen. Total RNA was extracted using TRI Reagent as per manufacturer's instructions (Sigma-Aldrich), followed by DNaseI treatment (Thermo Scientific). A total of $2 \mu \mathrm{g}$ of RNA was used for cDNA synthesis according to the manufacturer's instructions with the SuperScript II reverse transcriptase (Life Technologies). The PCR was carried out in a final reaction volume of $20 \mu \mathrm{L}$ using Maxima SYBR Green/ROX qPCR Master Mix (Thermo
Scientific) using the recommended conditions on a BioRad C1000 thermal cycler (BioRad). The PCR primers used were: PR1 (At2g14610) 5'-CGGAGCTA CGCAGAACAACT-3' and 5'CTCGCTAACCCACATG TTCA-3', UBQ10 (At4g05320) 5'-CACACTCCACTT GGTCTTGCGT-3' and 5'-TGGTCTTTCCGGTGAGAG TCTTCA-3'. The PCR program used for all reactions was: $95{ }^{\circ} \mathrm{C}$ for $10 \mathrm{~min}$, followed by 40 cycles consisting of $95{ }^{\circ} \mathrm{C}$ for $15 \mathrm{~s}, 55^{\circ} \mathrm{C}$ for $30 \mathrm{~s}$, and $72{ }^{\circ} \mathrm{C}$ for $30 \mathrm{~s}$. Each sample was assayed in triplicate and data were evaluated using BioRad CFX manager software, version 1.6 (BioRad). The relative PR1 expression was normalized using the $U B Q 10$ control and reported as fold increase in expression relative to the water treated control using the $\Delta \Delta \mathrm{Ct}$ method [50].

\section{Callose assay}

A. thaliana plants were pressure infiltrated with water alone, or water containing $10 \mu \mathrm{M}$ or $100 \mathrm{nM}$ of peptide. The leaves were harvested after $24 \mathrm{~h}$ of treatment and cleared with lactophenol. The callose deposits were stained with aniline blue and imaged by epifluorescent microscopy. The images were analyzed with ImageJ [51] software and the percentage of callose coverage in each image was calculated excluding staining of trichomes and the vasculature. A minimum of two images were taken from each of two leaves from two plants per treatment. The experiment was replicated four times with similar results.

\section{Seedling growth inhibition assay}

Seedlings of $A$. thaliana ecotype Col-0, xps1-1, or xps1-2 were grown for 5 days on MS-Agar plates prior to transfer of two seedlings to each well of a 24-well plate containing $400 \mu \mathrm{L}$ of $0.5 \times$ MS medium with $1 \%$ sucrose. The seedlings were treated with water or the noted dose of peptide and grown for a further 10-14 days. The seedlings were removed, briefly dried, and weighed (fresh weight). A minimum of four plants per genotype per treatment were included, and the experiment was replicated three times with similar results; all data are presented. For each replicate the mean fresh weight of the water control seedlings was calculated and used to normalize the weights of all seedlings of that genotype as a percentage of the control value.

\section{Virulence suppression assay}

A. thaliana plants were pressure infiltrated with water alone or water containing $10 \mu \mathrm{M}$ of peptide $24 \mathrm{~h}$ prior to infection with Pto DC3000 (0.0005 $\left.\mathrm{OD}_{600}\right)$. Bacterial growth was measured after $24 \mathrm{~h}$. Briefly, surface sterilized leaf disks were homogenized and the resulting culture was serially diluted and plated on solid selective media. The resulting colony counts were used to 
determine the number of colony forming units per surface area of the leaf. Each treatment was conducted on four leaves per plant of six individual plants. The experiment was repeated three times with similar results.

\section{XPS1 extracellular domain expression and purification}

For XPS1 expression in Hi5 cells, the ectodomain of XPS1 (36-650) was inserted into the baculovirus transfer vector pMelBac B (Invitrogen). The extracellular domain of XPS1 (amino acids 36-650) was cloned by ligation independent cloning between the existing Honey bee melettin signal sequence and the C-terminal Strep II-9x His tag. All clones were verified by Sanger Sequencing. Primers (Forward: GGT CGT ATA CAT TTC TTA CAT CTA TGC GAC GGA GGA GAC TGA TAA ACA AGC ATT GC; Reverse: GCA CCC TGG AAG TAC AGG TTC TCT GAT GAA TGC CTT CTT GGT AAT TCA ACA GAG C) were designed to have an amplification part homologous to the desired boundaries of XPS1 ectodomain and extensions for RecA-mediated SLIC strategy. The StrepII-9x His fused XPS1 ectodomain was produced by secreted expression in baculovirus-infected insect cells, harvested $72 \mathrm{~h}$ post infection and purified by Ni-NTA affinity chromatography (Qiagen). The samples were processed twice with a Superdex 200 16/60 column (GE Healthcare) pre-equilibrated with $10 \mathrm{mM}$ Bis-Tris pH 6.0, $150 \mathrm{mM} \mathrm{NaCl}$. Protein purity was checked by SDS-PAGE. The identity of the XPS1 ectodomain was further confirmed by anti-His immunoblots and mass spectrometry.

\section{MST binding assays}

The XPS1 ectodomain was kept at a constant concentration $(0.5 \mu \mathrm{M})$ in a buffer containing $10 \mathrm{mM}$ Bis-Tris pH6, $150 \mathrm{mM} \mathrm{NaCl}, 5$ glycerol and $0.01 \%$ Tween, whereas varying peptide concentrations were added. Approximately 4-6 $\mu \mathrm{L}$ of each sample was loaded in a fused silica capillary (NanoTemper Technologies). Measurements were performed at room temperature in a Monolith NT.label free instrument at a constant LED power of 20 and MST power of $60 \%$. Measurements were performed repeatedly on two independent protein preparations to ensure reproducibility. The data were analyzed by plotting xup 25 concentrations against percent changes of normalized fluorescence $(\Delta$ Fnorm $[\%] \mathrm{y}$ axis). Curve fitting was performed by using the NT affinity analysis software from Nanotemper.

\section{Luminol-based ROS generation assay}

Measurement of ROS production was performed using a modified version of the luminol-based assay as described [5]. Ten leaf discs (diameter $4 \mathrm{~mm}$ ) were taken from the leaves of 4-week-old Col-0 A. thaliana. Leaf disks were washed with $200 \mu \mathrm{L}$ of sterile water for $20 \mathrm{~h}$. The water was removed and replaced with $100 \mathrm{mM}$ Tris- $\mathrm{HCl}$
(pH 8.0) containing $20 \mu \mathrm{g} / \mathrm{mL}$ horseradish peroxidase and $34 \mu \mathrm{g} / \mathrm{mL}$ luminol. Wells were treated with water control or the noted dose of peptide. The luminescence was summed over a 2-s interval every $2 \mathrm{~min}$ for a period of $60 \mathrm{~min}$ on an Infinite M1000Pro microplate reader (TECAN Group Ltd.). Each treatment was performed on six wells.

\section{Sequence alignment and sequence logo}

The protein sequence of the xup25 containing xanthine uracil permease gene from each species used was extracted and aligned using MUSCLE [41]. The alignment was uploaded to the Gene Slider module of the BioAnalytical Resource for Plant Biology (bar.utoronto.ca) to produce the sequence logo.

\section{Ethics approval}

No ethics approval was necessary for this study.

\section{Data availability}

All of the genomic data produced for this study have been submitted to NCBI; the BioProject Accession numbers for these genomes along with all the publicly available genomes used can be found in Additional file 1: Table S1. The RefSeq ID numbers for all LRR-RLK sequences used can be found in Additional file 1: Table S5.

\section{Additional files}

Additional file 1: Contains Supplementary Tables S1-S5. (DOCX 56 kb)

Additional file 2: Figure S1. Comparison of peroxidase- and luminolbased PTI assays. Leaf disks from A. thaliana ecotype Col-0 plants were treated with water or the noted dose of flg22 peptide. Graphs are data from a single representative experiment. a Total POX activity was measured $20 \mathrm{~h}$ after treatment $\left(n=6,{ }^{* * *} P<0.001\right.$, pairwise Student's t-test, corrected with Holm-Bonferroni). b ROS production was quantified using luminol and horseradish peroxidase with luminescence measured for $2 \mathrm{~s}$ every $2 \mathrm{~min}$ for $60 \mathrm{~min}$ and the total summed. $\left(n=6,{ }^{* * *} P<0.001\right.$, pairwise Student's t-test, corrected with Holm-Bonferroni). Boxes show the lower quartile value, median value, and upper quartile value, while the whiskers extend to the lowest and highest values. (PNG $216 \mathrm{~kb}$ )

Additional file 3: Figure S2. Negative control peptides without predicted positively selected sites do not cause increased POX activity. Leaf disks from A. thaliana ecotype Col-0 plants were treated with water or $1 \mu \mathrm{M}$ of the indicated negative control peptide and total POX activity was measured $20 \mathrm{~h}$ after treatment. The mean value was calculated for each experimental replicate $(n=6)$ performed and these means plotted as data points on the graph. Each peptide was tested independently eight or nine times. Boxplot layout is as described in Fig. 1. (PNG 3 kb)

Additional file 4: Figure S3. flg22 does not cause increased POX activity in Arabidopsis ecotype Ws. Leaf disks from A. thaliana ecotype Ws plants were treated with water or $1 \mu \mathrm{M}$ of flg22 peptide. Total POX activity was measured $20 \mathrm{~h}$ after treatment. The experiment was replicated three times for a total $n=18$. Boxplot layout is as described in Fig. 1. (PNG 3 kb)

Additional file 5: Figure S4. xup25 does not induce POX activity in an independent xps 1 T-DNA insertion line: xps 1-2. Leaf disks from A. thaliana xps $1-2$ plants were treated with water or $1 \mu \mathrm{M}$ of peptide and total POX 
activity was measured $20 \mathrm{~h}$ after treatment. The mean value was calculated for each experimental replicate $(n=6)$ performed and these means plotted as data points on the graph. Each peptide was tested a total of four times except xup25 which was tested six times. Boxplot layout is as described in Fig. 1. (PNG 4 kb)

Additional file 6: Figure S5. Confirmation of T-DNA insertions for two independent xps 1 T-DNA insertion lines. a T-DNA insertion position for xps 1-1 and xps 1-2. Boxes represent exons and lines represent introns. $\mathbf{b}$ RT-PCR analysis of XPS1 expression in Col-0, xps 1-1, and xps 1-2 plants, respectively. The ubiquitously expressed UBQ10 is shown as a control for cDNA concentration. (PNG $51 \mathrm{~kb}$ )

Additional file 7: Figure S6. xup25 does not induce callose deposition in an independent xps 1 T-DNA insertion line: xps 1-2. Leaves from $A$. thaliana xps 1-2 plants were pressure infiltrated with water or $10 \mu \mathrm{M}$ of xup25, 100 nM xup25, or flg22 peptide. After $24 \mathrm{~h}$ of treatment the leaves were harvested, cleared, and callose deposits were stained prior to epifluorescent microscopy. The proportion of the image with callose present was determined $\left(n=6,{ }^{*} P<0.05,{ }^{* *} P<0.01\right.$, pairwise Student's $t$-test, corrected with Holm-Bonferroni). No significant difference was observed between Col-0, xps 1-1, and xps 1-2 plants treated with flg22. (PNG $242 \mathrm{~kb}$ )

Additional file 8: Figure S7. XPS1 binds specifically to xup25. Additional replicates of XPS1 binding assays. Titration of increasing amounts of xup25 peptide (a), but not flg22 (b), to a constant amount of XPS1 ECD $(0.5 \mu \mathrm{M})$ induces a significant MST signal shift. At each peptide concentration a measurement was made using $60 \%$ MST power and the values used to determine the $K_{D}$ or the EC50 with the NT affinity analysis software from Nanotemper. The $K_{D}$ fit plots are shown and both the $K_{D}$ and EC50 values are noted wherever possible. The calculated EC50 and $K_{D}$ values for xup25 were EC50 of $0.381 \mu \mathrm{M}$ and $K_{D}$ of $0.111 \mu \mathrm{M}$. The flg22 binding data could not be fitted using the EC50 fit and has a $K_{D}$ of $13.13 \mu \mathrm{M}$. The standard error of the regression fit is 3.8404566 for (a) and 6.4503735 for (b). (PNG $20 \mathrm{~kb}$ )

Additional file 9: Figure S8. Diversity of xup25 sequence across $P$. syringae species. a The protein sequences of the xup25 peptide from each species used in this study aligned. $\mathbf{b}$ The protein sequence of the xanthine uracil permease gene was aligned and used to produce a sequence logo, the xup25 sequence logo is marked and expanded below showing the diversity present in the peptide sequence. (PNG $1736 \mathrm{~kb}$ )

\section{Competing interests}

The authors declare that they have no competing interests.

\section{Authors' contributions}

GAM, ST, ES, PWW, YB, DD, and DSG designed experiments. GAM, ST, ES, PWW, and DSG performed experiments. GAM, ST, ES, PWW, YB, DD, and DSG wrote and edited the manuscript. All authors read and approved the final manuscript.

\section{Acknowledgements}

We would like to thank the members of the DSG and DD labs for helpful discussion, and in particular Sarah Cao and Alina Guna for assistance in plant maintenance. We thank the ABRC for T-DNA insertion lines used in this study. We thank the VBCF Protein Technologies Facility for excellent assistance.

\section{Funding}

This work was supported by Natural Sciences and Engineering Research Council of Canada Discovery Awards to DSG and DD; a Canada Research Chair in Plant-Microbe Systems Biology (DD) or Comparative Genomics (DSG); the Centre for the Analysis of Genome Evolution and Function (DD and DSG). This work was also supported by funds from the Austrian Academy of Science through the Gregor Mendel Institute (YB).

\section{Author details}

${ }^{1}$ Department of Cell \& Systems Biology, University of Toronto, 25 Willcocks St., Toronto, Ontario, Canada. ${ }^{2}$ Gregor Mendel Institute (GMI), Austrian Academy of Sciences, Vienna Biocenter (VBC), Dr Bohr Gasse 3, Vienna 1030, Austria. ${ }^{3}$ Centre for the Analysis of Genome Evolution \& Function, University of Toronto, Toronto, Ontario, Canada.
Received: 29 June 2015 Accepted: 14 April 2016

Published online: 09 May 2016

\section{References}

1. Dodds PN, Rathjen JP. Plant immunity: towards an integrated view of plantpathogen interactions. Nat Rev Genet. 2010;11:539-48.

2. Mott GA, Middleton MA, Desveaux D, Guttman DS. Peptides and small molecules of the plant-pathogen apoplastic arena. Front Plant Sci. 2014;5:677.

3. McCann HC, Nahal H, Thakur S, Guttman DS. Identification of innate immunity elicitors using molecular signatures of natural selection. Proc Natl Acad Sci U S A. 2012;109:4215-20.

4. Sun W, Dunning FM, Pfund C, Weingarten R, Bent AF. Within-species flagellin polymorphism in Xanthomonas campestris pv campestris and its impact on elicitation of Arabidopsis FLAGELLIN SENSING2-dependent defenses. Plant Cell. 2006;18:764-79.

5. Felix G, Duran JD, Volko S, Boller T. Plants have a sensitive perception system for the most conserved domain of bacterial flagellin. Plant J. 1999; 18:265-76.

6. Kunze G, Zipfel C, Robatzek S, Niehaus K, Boller T, Felix G. The N terminus of bacterial elongation factor Tu elicits innate immunity in Arabidopsis plants. Plant Cell. 2004;16:3496-507.

7. Monaghan J, Zipfel C. Plant pattern recognition receptor complexes at the plasma membrane. Curr Opin Plant Biol. 2012;15:349-57.

8. Greeff C, Roux M, Mundy J, Petersen M. Receptor-like kinase complexes in plant innate immunity. Front Plant Sci. 2012;3:209.

9. Shiu S-H, Bleecker AB. Receptor-like kinases from Arabidopsis form a monophyletic gene family related to animal receptor kinases. Proc Natl Acad Sci U S A. 2001;98:10763-8.

10. Wang G, Ellendorff U, Kemp B, Mansfield JW, Forsyth A, Mitchell K, et al. A genome-wide functional investigation into the roles of receptor-like proteins in Arabidopsis. Plant Physiol. 2008;147:503-17.

11. Gou X, He K, Yang H, Yuan T, Lin H, Clouse SD, et al. Genome-wide cloning and sequence analysis of leucine-rich repeat receptor-like protein kinase genes in Arabidopsis thaliana. BMC Genomics. 2010;11:19.

12. Sun Y, Li L, Macho AP, Han Z, Hu Z, Zipfel C, et al. Structural basis for flg22induced activation of the Arabidopsis FLS2-BAK1 immune complex. Science. 2013;342:624-8

13. Schulze B, Mentzel T, Jehle AK, Mueller K, Beeler S, Boller T, et al. Rapid heteromerization and phosphorylation of ligand-activated plant transmembrane receptors and their associated kinase BAK1. J Biol Chem. 2010;285:9444-51.

14. Hou S, Wang $X$, Chen D, Yang $X$, Wang M, Turrà D, et al. The secreted peptide PIP1 amplifies immunity through receptor-like kinase 7. PLoS Pathog. 2014;10:e1004331.

15. Minibayeva F, Beckett RP, Kranner I. Roles of apoplastic peroxidases in plant response to wounding. Phytochemistry. 2015;112:122-9.

16. Daudi A, Cheng Z, O'Brien JA, Mammarella N, Khan S, Ausubel FM, et al. The apoplastic oxidative burst peroxidase in Arabidopsis is a major component of pattern-triggered immunity. Plant Cell. 2012;24:275-87.

17. Mammarella ND, Cheng Z, Fu ZQ, Daudi A, Bolwell GP, Dong X, et al. Apoplastic peroxidases are required for salicylic acid-mediated defense against Pseudomonas syringae. Phytochemistry. 2015;112:110-21.

18. Chinchilla D, Bauer Z, Regenass M, Boller T, Felix G. The Arabidopsis receptor kinase FLS2 binds flg22 and determines the specificity of flagellin perception. Plant Cell. 2006;18:465-76.

19. Gómez-Gómez L, Boller T. FLS2: an LRR receptor-like kinase involved in the perception of the bacterial elicitor flagellin in Arabidopsis. Mol Cell. 2000;5:1003-11.

20. Zipfel C, Kunze G, Chinchilla D, Caniard A, Jones JDG, Boller T, et al. Perception of the bacterial PAMP EF-Tu by the receptor EFR restricts Agrobacterium-mediated transformation. Cell. 2006;125:749-60.

21. Song WY, Wang GL, Chen LL, Kim HS, Pi LY, Holsten T, et al. A receptor kinase-like protein encoded by the rice disease resistance gene, Xa21. Science. 1995;270:1804-6.

22. Holton N, Nekrasov $V$, Ronald PC, Zipfel C. The phylogenetically-related pattern recognition receptors EFR and XA21 recruit similar immune signaling components in monocots and dicots. PLoS Pathog. 2015;11: e1004602.

23. Bahar O, Pruitt R, Luu DD, Schwessinger B, Daudi A, Liu F, et al. The Xanthomonas Ax21 protein is processed by the general secretory system and is secreted in association with outer membrane vesicles. PeerJ. 2014;2:e242. 
24. Pruitt RN, Schwessinger B, Joe A, Thomas N, Liu F, Albert M, et al. The rice immune receptor XA21 recognizes a tyrosine-sulfated protein from a Gramnegative bacterium. Sci Adv. 2015;1:e1500245-5.

25. Gómez-Gómez L, Felix G, Boller T. A single locus determines sensitivity to bacterial flagellin in Arabidopsis thaliana. Plant J. 1999;18:277-84.

26. Asai T, Tena G, Plotnikova J, Willmann MR, Chiu W-L, Gomez-Gomez L, et al. MAP kinase signalling cascade in Arabidopsis innate immunity. Nature. 2002:415:977-83.

27. Zipfel C, Robatzek S, Navarro L, Oakeley EJ, Jones JDG, Felix G, et al. Bacterial disease resistance in Arabidopsis through flagellin perception. Nature. 2004; 428:764-7.

28. Seidel SAl, Dijkman PM, Lea WA, van den Bogaart G, Jerabek-Willemsen M, Lazic A, et al. Microscale thermophoresis quantifies biomolecular interactions under previously challenging conditions. Methods. 2013;59:301-15.

29. Clarke CR, Chinchilla D, Hind SR, Taguchi F, Miki R, Ichinose $Y$, et al. Allelic variation in two distinct Pseudomonas syringae flagellin epitopes modulates the strength of plant immune responses but not bacterial motility. New Phytol. 2013;200:847-60.

30. Naito K, Taguchi F, Suzuki T, Inagaki Y, Toyoda K, Shiraishi T, et al. Amino acid sequence of bacterial microbe-associated molecular pattern flg22 is required for virulence. Mol Plant Microbe Interact. 2008;21:1165-74.

31. Nekrasov V, Li J, Batoux M, Roux M, Chu Z-H, Lacombe S, et al. Control of the pattern-recognition receptor EFR by an ER protein complex in plant immunity. EMBO J. 2009;28:3428-38.

32. Cai R, Lewis J, Yan S, Liu H, Clarke CR, Campanile F, et al. The plant pathogen Pseudomonas syringae pv. tomato is genetically monomorphic and under strong selection to evade tomato immunity. PLoS Pathog. 2011; 7:e1002130.

33. O'Brien HE, Thakur S, Guttman DS. Evolution of plant pathogenesis in pseudomonas syringae: a genomics perspective. Annu Rev Phytopathol. 2011:49:269-89.

34. O'Brien HE, Thakur S, Gong Y, Fung P, Zhang J, Yuan L, et al. Extensive remodeling of the Pseudomonas syringae pv. avellanae type III secretome associated with two independent host shifts onto hazelnut. BMC Microbiol. 2012;12:141

35. Belkhadir $Y$, Yang L, Hetzel J, Dangl JL, Chory J. The growth-defense pivot: crisis management in plants mediated by LRR-RK surface receptors. Trends Biochem Sci. 2014;39:447-56.

36. Li J, Wen J, Lease KA, Doke JT, Tax FE, Walker JC. BAK1, an Arabidopsis LRR receptor-like protein kinase, interacts with BRI1 and modulates Brassinosteroid signaling. Cell. 2002;110:213-22.

37. Nam KH, Li J. BRI1/BAK1, a receptor kinase pair mediating Brassinosteroid signaling. Cell. 2002;110:203-12.

38. Bauer Z, Gómez-Gómez L, Boller T, Felix G. Sensitivity of different ecotypes and mutants of Arabidopsis thaliana toward the bacterial elicitor flagellin correlates with the presence of receptor-binding sites. J Biol Chem. 2001; 276:45669-76.

39. Danna CH, Millet YA, Koller T, Han S, Bent AF, Ronald PC, et al. The Arabidopsis flagellin receptor FLS2 mediates the perception of Xanthomonas Ax21 secreted peptides. Proc Natl Acad Sci U S A. 2011;108: 9286-91.

40. Li L, Stoeckert CJ, Roos DS. OrthoMCL: identification of ortholog groups for eukaryotic genomes. Genome Res. 2003;13:2178-89.

41. Edgar RC. MUSCLE: a multiple sequence alignment method with reduced time and space complexity. BMC Bioinformatics. 2004;5:113.

42. Abascal F, Zardoya R, Telford MJ. TranslatorX: multiple alignment of nucleotide sequences guided by amino acid translations. Nucleic Acids Res. 2010;38:W7-13.

43. Price MN, Dehal PS, Arkin AP. FastTree: computing large minimum evolution trees with profiles instead of a distance matrix. Mol Biol Evol. 2009;26:1641-50.

44. Yang Z. PAML 4: phylogenetic analysis by maximum likelihood. Mol Biol Evol. 2007:24:1586-91.

45. Boratyn GM, Schäffer AA, Agarwala R, Altschul SF, Lipman DJ, Madden TL. Domain enhanced lookup time accelerated BLAST. Biol Direct. 2012:7:12.

46. Altschul SF, Madden TL, Schäffer AA, Zhang J, Zhang Z, Miller W, et al. Gapped BLAST and PSI-BLAST: a new generation of protein database search programs. Nucleic Acids Res. 1997;25:3389-402.

47. Katoh K, Kuma K, Toh H, Miyata T. MAFFT version 5: improvement in accuracy of multiple sequence alignment. Nucleic Acids Res. 2005;33:511-8.

48. Tamura K, Peterson D, Peterson N, Stecher G, Nei M, Kumar S. MEGA5: molecular evolutionary genetics analysis using maximum likelihood, evolutionary distance, and maximum parsimony methods. Mol Biol Evol. 2011;28:2731-9.

49. Alonso JM, Stepanova AN, Leisse TJ, Kim CJ, Chen H, Shinn P, et al. Genome-wide insertional mutagenesis of Arabidopsis thaliana. Science. 2003;301:653-7.

50. Livak KJ, Schmittgen TD. Analysis of relative gene expression data using real-time quantitative PCR and the 2(-Delta Delta C(T)) Method. Methods. 2001;25:402-8.

51. Schneider CA, Rasband WS, Eliceiri KW. NIH Image to ImageJ: 25 years of image analysis. Nat Methods. 2012;9:671-5.

\section{Submit your next manuscript to BioMed Central and we will help you at every step:}

- We accept pre-submission inquiries

- Our selector tool helps you to find the most relevant journal

- We provide round the clock customer support

- Convenient online submission

- Thorough peer review

- Inclusion in PubMed and all major indexing services

- Maximum visibility for your research

Submit your manuscript at www.biomedcentral.com/submit 